\title{
QRishing: The Susceptibility of Smartphone Users to QR Code Phishing Attacks
}

\author{
Timothy Vidas, Emmanuel Owusu, Shuai Wang, Cheng Zeng, \\ Lorrie Faith Cranor, and Nicolas Christin \\ Carnegie Mellon University \\ Pittsburgh, PA, USA \\ \{tvidas, eowusu, shuaiwang, chengzeng, lorrie, nicolasc\}@cmu.edu
}

\begin{abstract}
The matrix barcodes known as Quick Response (QR) codes are rapidly becoming pervasive in urban environments around the world. QR codes are used to represent data, such as a web address, in a compact form that can be scanned readily and parsed by consumer mobile devices. They are popular with marketers because of their ease in deployment and use. However, this technology encourages mobile users to scan unauthenticated data from posters, billboards, stickers, and more, providing a new attack vector for miscreants. By positioning QR codes under false pretenses, attackers can entice users to scan the codes and subsequently visit malicious websites, install programs, or any other action the mobile device supports. We investigated the viability of QRcode-initiated phishing attacks, or QRishing, by conducting two experiments. In one experiment we visually monitored user interactions with QR codes; primarily to observe the proportion of users who scan a QR code but elect not to visit the associated website. In a second experiment, we distributed posters containing QR codes across 139 different locations to observe the broader application of QR codes for phishing. Over our four-week study, our disingenuous flyers were scanned by 225 individuals who subsequently visited the associated websites. Our survey results suggest that curiosity is the largest motivating factor for scanning QR codes. In our small surveillance experiment, we observed that $85 \%$ of those who scanned a QR code subsequently visited the associated URL.
\end{abstract}

Keywords: Phishing, Mobile, Security, QR Code, Smartphone

\section{Introduction}

A Quick Response code (QR code) is a two-dimensional matrix of black and white pixels [20] that can be used to store information in a compact and opticallyscannable form. QR codes have gained popularity due to their higher information density and improved readability compared to one-dimensional barcodes. As the number of smartphone users grows rapidly [8], businesses are turning to QR codes en masse to provide a fun and simple way to direct smartphone users to their websites and products. QR codes are designed to be readable regardless of orientation and in cases where a code is partially damaged or masked. These properties facilitate the use of $\mathrm{QR}$ codes in consumer applications to convey information to users. 


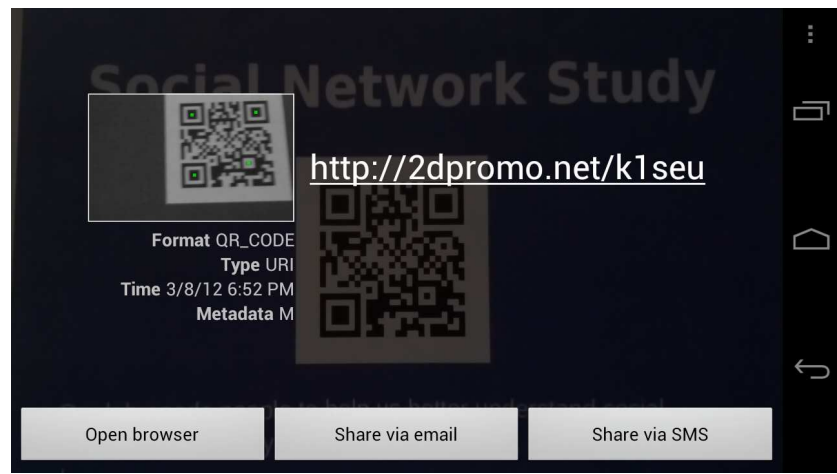

Fig. 1: Screen capture of the most popular "barcode scanner" on Android: ZXing. With default settings, the URL is prominently shown to the user after scanning.

QR codes are typically "scanned" by photographing the QR code using a mobile device, such as a smartphone. The image is then interpreted by a QR code reader that users may install as an application on their mobile device. The reader decodes the message and performs an operation based on the message. For example, if the encoded data contains a link to a mobile application download, the reader may launch a marketplace application such as Google Play or Apple App Store. The content represented by a QR code is often a hyperlink, and the associated action is to launch the device's web browser and visit the website specified by the code.

QR codes can be found on store-front windows, magazines, newspapers, websites, posters, mass mailings, and billboards. Businesses display QR codes on advertisements to direct people to their websites. One study found over 14 million U.S. mobile users scanning QR codes during June 2011 [26].

The ease with which one can create and distribute QR codes has not only attracted businesses, but also scammers seeking to direct people to phishing websites. Phishing is a semantic attack that cons individuals, under the guise of a legitimate organization or individual, into visiting a malicious website or providing sensitive information [21]. With the increased usage of QR codes, QR code phishing, or QRishing (phonetically: "krihsh-ing"), presents a threat to this new, convenient technology. Concerns for the safety of QR codes are increasing $[11,24,26,28]$. An attacker might place a sticker of a QR code containing malicious content over a legitimate QR code or create an entirely new $\mathrm{QR}$ code advertisement masquerading as a legitimate entity.

Some QR code reader applications may perform actions without first presenting the human-readable QR code content to the user. For example, an application may automatically open a hyperlink in the device's web browser without permitting the user to first verify the hyperlink. In this case, it is easier for attackers to deceive users into divulging private information or, even worse, installing malicious software on their phones. On the other hand, if the barcode application displays the URL to the user, an astute user may notice a suspicious-looking URL. However, use of "URL shorteners" can make it more difficult for users to 
evaluate a URL. Figure 1 depicts an application displaying the URL to the user and awaiting further action by the user.

To frame the scope of the problem, we tested the most popular reader applications from the Android and Apple marketplaces in January 2012. We downloaded and tested the top ten free applications for "barcode scanner" from Google Play and the Apple App Store. Thirty percent of these top ten free scanning applications in the Google Play Market and 50\% in the Apple App Store immediately visit a scanned URL in the default configuration. Tables showing the particular applications and results can be found in the appendix.

The purpose of this study is to measure the threat QR codes pose as a phishing attack vector and to identify ways to improve the safety of QR code interaction. We are interested in the behaviors of smartphone users when they see QR codes posted in public places, including whether or not they look for context around the QR code, scan the QR code, and visit the website from the QR code.

We further motivate the problem with related work in Section 2. The user study consisted of two experiments: (1) A QRishing experiment and (2) a baseline surveillance study of user interaction with $\mathrm{QR}$ codes, which we describe in Section 3 and Section 4, respectively. Security implications of the study are presented in Section 5 and conclusions in Section 6.

\section{Related Work}

Phishing is a type of semantic attack where the malicious party attempts to gain sensitive information (e.g., account credentials or credit card numbers) by baiting victims with communications and content that appears to be from a legitimate party (e.g., a counterfeit password change website). Existing research has repeatedly shown that typical computer users have difficulty distinguishing between legitimate content and phishing content [14-16,32]. QRishing is an extension of phishing that utilizes QR codes.

Downs et al. interviewed 20 non-expert computer users about their decisionmaking process when they encountered suspicious looking emails [16]. Their study suggests that simply being aware of Phishing-style scams is insufficient. Furthermore, their findings suggest that providing message-specific contextual cues (e.g., "this website is requesting a password") may be more effective than sender-specific cues, as scammers exploit the fact that many victims have a real account with the entity that is being faked.

The economic viability of typosquatting demonstrates the usefulness of misspelled and misleading domain names [22]. The only technological controls currently available to counter QRishing rely on the user to identify questionable URLs or involve cues from external tools, such as domain blacklisting services. Such cues have previously been shown to be misunderstood by users [17] who may not even understand the difference between positive and negative cues [14].

Dhamija et al. found that nearly a quarter of their 22 participants did not use browser-based cues (e.g., the address bar and status bar) leading to incorrect identification of fraudulent websites [15]. We find similar results, in the case 
where QR codes are used as a medium for phishing - specifically, 36\% of our phishing participants indicated that they did not or could not recall checking the link.

The ease with which one can access web content via QR codes may induce more users to ignore browser-based cues as compared to entering or following a link. A typical QR code use case involves a scan and a click. Perceptive and security-conscious users may pause to examine the hyperlink but, in general, there is very little explicit interaction with the encoded data. The use of shortened URLs and the limited screen space of smartphones further obscures browser-based cues.

Similar to other phishing work, non-technical controls, such as increasing user education [27], may produce similar effects when applied to QRishing. A combination of automated detection systems along with user education may prove to be the best approach [21].

The security and usability research communities have explored various proposals for combating phishing attacks. Zhang et al. leveraged message-specific contextual cues for automated phishing detection in their implementation of CANTINA [32]. Dhamija et al. presented a defense against phishing attacks that made use of trusted paths to prevent window spoofing and independently computed images that allowed users to authenticate the remote party by visually verifying that the expected image was received [14].

Phishing and the effects of malware are perhaps more threatening on mobile devices than on traditional computers. The management model, long patching cycle, limited screen space, and myriad of input types and sensors found on mobile devices make mobile oriented malware particularly distressing $[19,30]$.

Concurrent to our research, an Internet vigilante claimed to have conducted a QRishing attack by changing his Twitter icon to a $\mathrm{QR}$ code. This QR code represented a shortened URL that ultimately led victims to a webpage that reportedly hosted a WebKit browser exploit and secondary exploits for iOS and Android devices. This five-day attack is claimed to have garnered 1200 victims of which 500 successfully executed the secondary OS-specific payload [31].

In addition to the claimed QRishing attack via Twitter, industry researcher Eric Mikulas has recently presented work on QR code phishing attacks [29]. Following our study, Mikulas conducted a similar attack predominately using small stickers to place QR codes around Pittsburgh, PA. Similar to our study, these QR codes lead smartphone users to an informative website about QR code risks. After posting 80 stickers and receiving fewer website visits than expected, Mikulas said he plans to "attach them to fliers offering a false incentive or even place his stickers on top of existing advertisements and QR codes."

\section{QRishing Experiment}

The ease with which one can create and distribute QR codes may make them attractive to scammers seeking to direct people to phishing websites. The purpose of this study is to understand how users interact with QR codes in public spaces and to assess the susceptibility of smartphone users to QRishing attacks. In this 


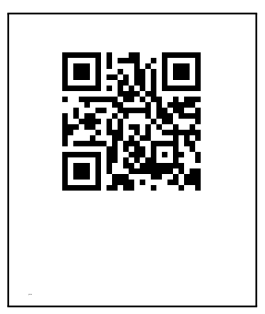

(a) qrcode_only

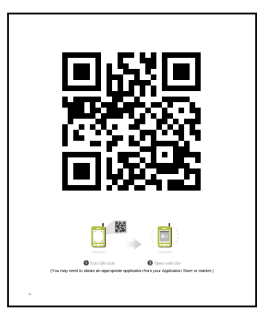

(b) qrcode_inst.

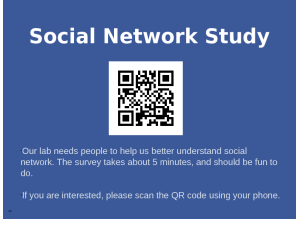

(c) qrcode_SNS

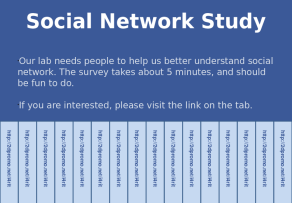

(d) ripoff_SNS

Fig. 2: An example flyer for each of the four conditions deployed in the QRishing experiment. (a) shows qrcode_only -flyers with a QR code. (b) shows qrcode_instructions -flyers with a QR code and usage instructions. (c) shows qrcode_SNS flyers advertising a mock SNS study with QR code. (d) shows ripoff_SNS -flyers advertising a mock SNS study with rip-off tabs.

experiment, we posted flyers around the city of Pittsburgh, PA. Each passerby who scanned one of the flyers was directed to a a brief online survey.

\subsection{Methodology}

We posted flyers with QR codes both on the Carnegie Mellon University campus and in public locations around Pittsburgh (e.g., at bus stops, public bulletin boards at restaurants, coffee shops, etc). All flyers were posted in public locations where flyers are routinely placed. Each QR code on a poster represented a unique URL to our webserver, allowing us to unambiguously know in which location the participant observed our flyer. We used random, unique URLs similar to popular "URL shortening" services for each flyer. Such URLs are commonly used in QR code advertising. Further, the use of random URLs minimizes the risk that after scanning one flyer, curious participants could easily determine and visit URLs associated with other flyers. In the last week of January and first week of February 2012, we posted flyers at 139 different locations: 104 campus locations, 35 off-campus locations. Each flyer was checked weekly and, if needed, replaced. This experiment had four conditions (pictured in Figure 2):

- qrcode_only. A flyer with only a QR code.

- qrcode_instructions. In addition to the QR code graphic, includes instructions on how to use a QR code.

- qrcode_SNS. Innocuous flyer utilizing a QR code (a "social networking" user study advertisement).

- ripoff_SNS. A user study flyer similar to 3, but with traditional rip-off tabs instead of a QR code.

All conditions were randomly distributed across the locations and ran simultaneously for four weeks. When a person scanned the QR codes (or visited the URL on the rip-off tab), they were taken to our website where they were informed about the experiment and prompted to participate in an optional survey.

Conditions qrcode_only and qrcode_instructions did not have any advertised function, thus any participant in these conditions is likely to have scanned the 


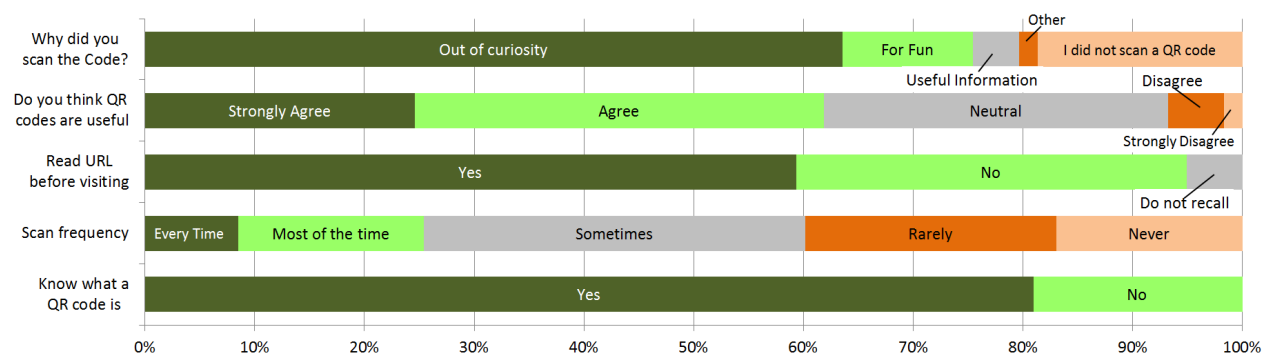

Fig. 3: Survey responses. Most participants scanned QR codes out of curiosity, agree than QR codes are useful, read the URL prior to visiting the website, and know the term "QR code."

QR code out of curiosity, compulsion, fun, etc. Conditions qrcode_only, qrcode_instructions and qrcode_SNS all involve the use of QR codes and thus provided insight into the frequency with which QR codes on flyers are scanned. Without a QR code, ripoff_SNS served as a performance baseline to compare with the other three conditions.

Regardless of condition, upon visiting the URL, participants were notified of the study via webpage and given the choice to follow a link to take an optional survey. We also recorded the access time, IP address, and user-agent from the server web log. Upon completion of the survey (or electing not to participate in the survey) the participant was automatically taken to a debrief webpage for the experiment. Participants who reported being under 18 years old were informed that their data would not be used in research and we discarded associated data.

\subsection{Results}

Of the 139 posted flyers, $85(61 \%)$ were utilized by participants to visit the study website at least once, totaling 225 hits across all conditions. Examination of source address, access time and poster location (URL) indicated that only once did the same device scan a QR code twice. One hundred twenty-two participants (54\%) completed the optional survey. Seventeen participants started, but did not complete the survey, and five participants self-reported to be under 18, and were removed from the study.

In the survey, participants were asked "Do you know what a QR code is?" The majority $(83 \%)$ of survey takers responded "Yes," indicating some familiarity with the technology. Even 51\% of participants in ripoff_SNS, which did not use a QR code, answered "Yes," further indicating that participants were aware of the technology. We posit that although some smartphone users may not know the term "QR code," the majority of users know the function of a QR code when presented with one.

We also asked participants about the primary reason they chose to scan the QR code (including an option for "I did not scan a QR code"). We observed far more participants scanning the QR code out curiosity than for related information. Figure 3 shows the distribution of survey responses from participants. More 


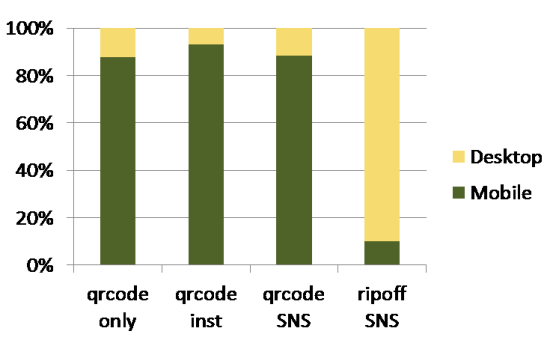

Fig. 4: Mobile vs desktop users by condition.

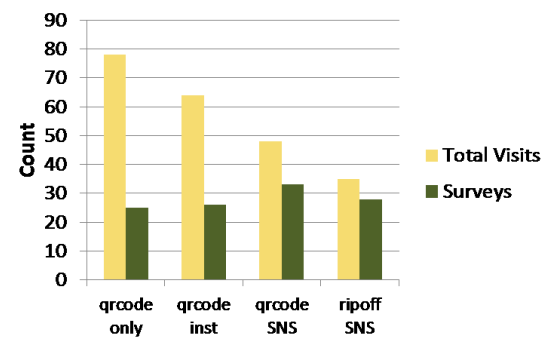

Fig. 5: Visited URLs and Survey Completion by condition

than $75 \%$ of the survey respondents scanned the flyer out of curiosity $(64 \%)$ or for fun (14\%). Less than $4 \%$ claim to have scanned the QR code because the related information seemed useful. Twenty percent of the respondents indicate that they did not scan a QR code, and all of these participants were in ripoff_SNS. As expected, participants not using a mobile device were also predominately in the condition without a QR code, ripoff_SNS, though not exclusively (Figure 4).

Among the four conditions, qrcode_only had the most participants while ripoff_SNS had the least number of participants. Figure 5 shows the distribution of participants who both visited the URL and completed the survey across the four conditions. While curiosity was reported to be the main reason for initially scanning a QR code, participants were significantly more likely $\left(\chi^{2}=8.7344\right.$, df $=1, \mathrm{p}=0.003)$ to complete the survey in conditions that explicitly advertised a study than those that had no advertised functionality.

Fifty-eight percent of respondents report reading the URL prior to visiting the link. While this behavior is likely safer than that of the $36 \%$ who did not read the URL, they still visited an obscure URL to an unrecognized domain (we registered the domain just prior to the study).

Across all four conditions we found that men were at least 2.5 times more likely to participate, especially in qrcode_only where we observed more than 7.6 times as many male participants. While we are uncertain of exactly how many individuals passed our flyers, nor how many of them possessed mobile devices, we can approximate percentages based on demographic data for the respective areas. For the on-campus flyers we can compare to CMU's general population [25], and for off-campus flyers we can compare to Pittsburgh census [12] data for the area we posted flyers. Further, we can use market penetration data [4] [5] to approximate percentages of smartphone owners. The gender distribution oncampus is $63 \%$ male, $37 \%$ female [25], and off-campus in the Pittsburgh area is $52 \%$ male, $48 \%$ female [12]. Among U.S. smartphone users, the gender is distributed $47 \%$ male, $53 \%$ female [5]. The incumbent population suggests that approximately $50-60 \%$ of our participants should be male, yet we observed $75 \%$.

The only condition that fell within the expected gender ratio was qrcode_instructions. As shown in Figure 6, qrcode_instructions has fewer male respondents and more "Prefer not to answer" than the other three conditions. There is no way to tell the gender of those who selected "Prefer not to answer." While the 


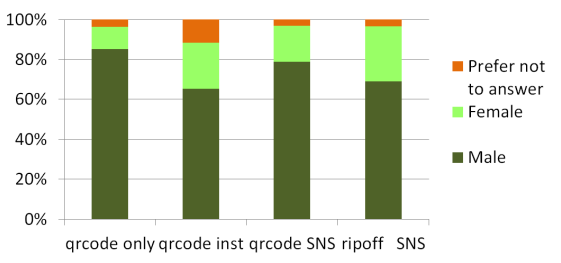

Fig. 6: Self-reported gender by condition. qrcode_instructions has fewer male and more "Prefer not to answer" participants than any other condition.

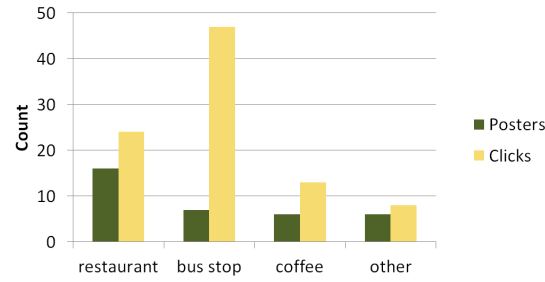

Fig. 7: Poster performance by location type. For each location type, green bars show the number of fliers posted, and yellow bars show accumulated clicks.

result is not statistically significant, it is clear that in our experiment qrcode_instructions had more respondents who wished not to reveal their gender.

Our two most observed age ranges (on and off campus) were 18-24 and 2534 , together accounting for $78 \%$ of our participants. This closely aligns with the two age groups that have most adopted smartphones [4].

We found that flyers at bus stops far outperformed other locations. On average, flyers posted at bus stops solicited nearly seven URL visits per flyer. Figure 7 shows the distribution of off-campus flyers as grouped by bus stop, restaurant, coffee shop and other. Flyers posted at bus stops may receive more attention simply due to behavior at such a location. For example, those waiting for the bus to arrive are likely bored and are forced to wait idly at the location for a non-trivial duration.

We examined other metrics such as day-of-week, time-of-day, and user perception of QR code usefulness, all of which did not prove useful as a predictor of behavior. Additionally, we examined the networks from which devices were connecting, and the results were as expected in the U.S. Of the cellular network users $54 \%$ used Verizon Wireless, $31 \%$ AT\&T, and $15 \%$ Sprint. The non-cellular users primarily $(63 \%)$ used campus networks while the primary home Internet providers where Comcast (18\%) and Verizon (6\%).

\subsection{Limitations}

Unlike the envisioned attack scenario, we are bound by ethical, legal, and Institutional Review Board limitations in the presentation and placement of QR codes. A would-be attacker may have little consideration for vandalism, covering existing QR codes with his own, or any number of other less scrupulous activities.

Like many on-campus studies, our observed population for on-campus flyers is biased to the local population of CMU. Similarly, our off-campus flyer locations were subject to the respective populations in Pittsburgh and may not be representative of other areas.

In qrcode_SNS and ripoff_SNS, the "social networking user study," the flyers will have only attracted individuals interested in such a study. Other false 
pretenses could have been employed, such as a local band or work-from-home opportunities, but would have similarly limited the set of individuals attracted to the flyer.

In our experiments we used "shortened URLs," which have their own security implications [23]. It is possible that users may be more likely to follow a typical URL, but we felt that using a shortened URL exhibited more realistic conditions as shortened URLs are often used in QR code advertisements. The short property of shortened URLs also fits nicely with mobile devices as the limited screen space will cause many URLs to be truncated for display, resulting in the user only having the ability to observe part of the URL. Further, we wanted seemingly random URLs so that users could not easily predict the URL of a poster they had not physically encountered.

Particular to ripoff_SNS, participants may have been less likely to correctly type or may have had less desire to participate in the study due to the URL format. The URLs used in all conditions were similar to those found in popular URL shortening services (e.g., http://bit.ly, http://goo.gl). Such a random pattern (e.g., skx0r132) may be perceived differently by participants than a link consisting of a domain name and a common webpage naming convention (e.g., study.php).

\section{Surveillance Experiment}

Since QR codes are abundant in urban areas, we wanted to observe how people interact with them in public spaces. Specifically, we wanted to identify how many participants would scan the QR code and also visit the associated website versus the number of participants who would scan the QR code but elect not to visit the website. This observation provides insight about the potential for QR codes as a phishing attack vector because examining the URL is a practical and effective defense against many phishing threats. The use of $\mathrm{QR}$ codes minimize the person's effort in obtaining a URL; the person does not have to manually transcribe the URL from the source material. Such reduced interaction may encourage the unsafe behavior of visiting a questionable website without seeing the URL, sacrificing security in favor of usability.

This section describes the methodology, experimental design, and analysis of the surveillance experiment. We refer to this experiment as surveillance_exp in the subsequent text.

\subsection{Methodology}

We posted a flyer containing a QR code on a bulletin board at Carnegie Mellon University and placed it under video surveillance. By comparing captured video footage of people scanning the $\mathrm{QR}$ code with server logs, we were able to identify the number of participants who scanned the code as well as the number of participants who actually visited the URL encoded in the QR code. If a corresponding entry did not exist on the server we assumed that the participant scanned the QR code, but chose not to visit the website. The experiment had two conditions: an incentive condition and a no-incentive condition. 
- surv_qrcode_only. In the no-incentive case, we collected two weeks of footage using a flyer containing only a QR code, similar to Figure 2(a).

- surv_incentive. Following the incentive case, we collected two additional weeks of footage using a flyer offering the chance to win a $\$ 50$ Amazon gift card.

The sequence of events a participant followed in both conditions is as follows. First, a person who walks by the bulletin board noticed our flyer. They became a participant in our study when they entered the field of view of the camera and scanned the QR code. If the participant chose to visit the website (or the reader application automatically opened the link), they were presented with a simple web page that thanked the person for their interest in the study and asked them to take a survey. The person may have selected "continue" to further participate by taking an online survey, selected "cancel" to continue directly to the debrief material, or simply elected to close the browser. Similar to the qrishing_exp experiment, participants who reported that they were under the age of 18 received an additional debrief message stating that their data would not be used in the study and that they were not eligible to receive the incentive.

Every time a participant accessed our secure server, we recorded the time of access, the IP address, and user-agent in the web server log. The IP address was used to assess the connection type (e.g., campus Wi-Fi). Participants in surv_incentive were asked to provide their email address in order to be notified in the event they won the gift card. Providing an email address was at the sole discretion of participant. Furthermore, we ensured that a participant's email was not correlated with her survey responses.

\subsection{Data Capture}

We posted a flyer containing a QR code in the Gates Center for Computer Science on CMU's Pittsburgh campus. The flyer was posted on an announcements board located on the main floor of the building, an area which is open to the public and access-controlled only at night. A camera and netbook were mounted above the board to capture the activity of people around the poster. We checked the flyer daily to ensure it was unobstructed and that there were no other QR codes nearby. After some field trials at the site using both Android and iPhone smartphones, we concluded that a 3-by-3 inch QR code would best ensure the participant was within the field of view of the camera.

The netbook was configured to capture data only when motion was detected. The camera recorded four frames per second for as long as there was motion, and for 60 seconds thereafter. Each time a picture was captured, it was immediately processed with an edge-detection algorithm in order to minimize storing more data than required by the experiment and to protect the privacy of the participant. The experiment configuration is depicted in Figure 8.

An example of data captured from one participant's interaction is shown in Figure 9. This figure shows a participant approaching the flyer, photographing the flyer, and leaving the experiment area. 


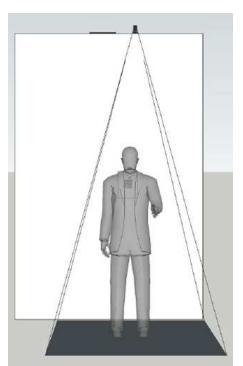

(a) Front

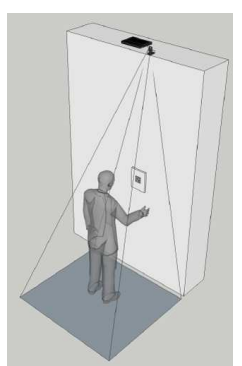

(b) Above

Fig. 8: Surveillance experiment equipment configuration. The camera and netbook are mounted on the area above the announcements board. The box around the person represents the field of view of the camera. (a) shows the setup from the front, facing the board. (b) shows an isometric view from above.

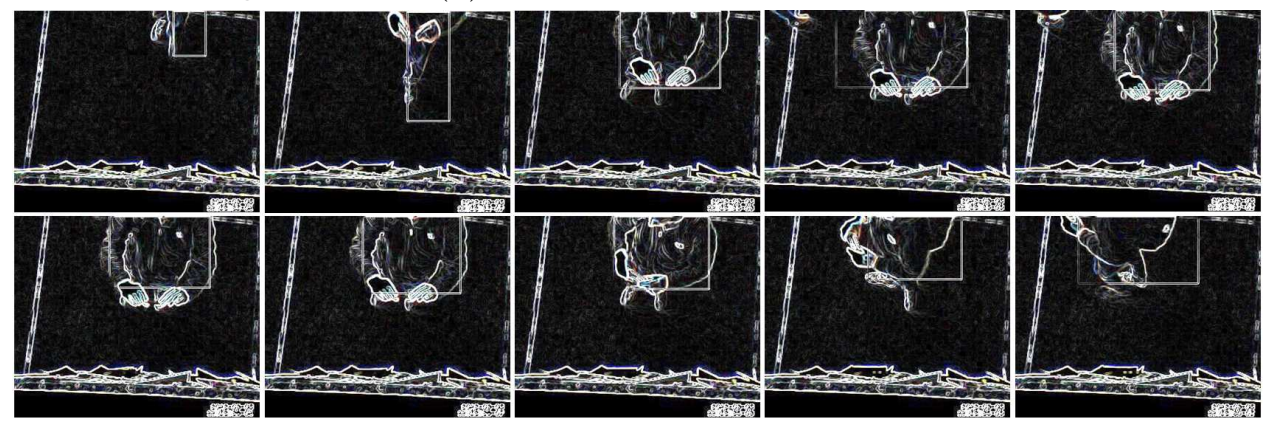

Fig. 9: A participant photographing the flyer posted on the bulletin board at the bottom of each frame. The sequential progression is from left-to-right and topto-bottom. The rectangles that overlay the participant indicate software motion detection. Each frame is processed using an edge-detection algorithm in order to minimize capturing more data than required by the experiment and to protect the privacy of the participant.

\subsection{Removing False Positives}

Due to the sensitivity of the software motion detection and the communal nature of the experiment site, the vast majority of collected images are not imminently useful. In many cases, passersby will briefly trigger data capture, people will move chairs into the field-of-view or otherwise congregate or loiter. Our flyer was secured at each corner with thumb-tacks, however other flyers may have been secured only at the top leading to some circumstances where activity outside of camera view caused flyers to move. The situation most apt to provide a false positive is when a subject appears to be facing the flyer, but it is not clear if the subject is actually photographing the flyer. By examining such situations in context with time-adjacent images, we are able to identify unrelated activities, such as posting or retrieving a flyer unrelated to our study. Examples of each of these false positives are provided in Figure 10. The captured images provide enough fidelity to accurately determine which should be discarded from analysis. 


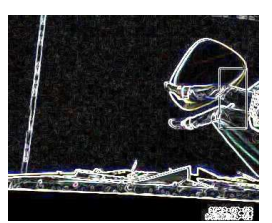

(a) Foreign object

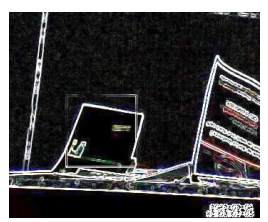

(b) External effects (wind)

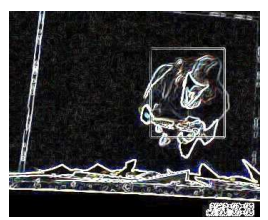

(c) Possible participant

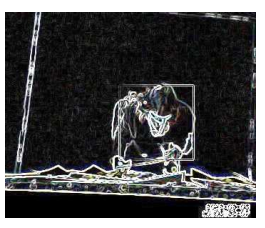

(d) Posting an unrelated flyer

Fig. 10: The communal nature of the experiment site encourages inhabitants to loiter, as seen in (a) where an individual has moved a chair into the experiment area and relaxed. (b) depicts nearby "wind" which occasionally caused nearby flyers to trigger motion detection. From the frame shown in (c), it is difficult to discern if the person in is scanning the flyer or not. However with the additional context of time-adjacent frames, it is obvious that the person is searching for the best location to post a flyer unrelated to the study. The posting, (d), is performed several seconds after (c).

\subsection{Results}

We collected data for four weeks beginning February 7, 2012, two weeks using the surv_qrcode_only display (10 participants) followed by two weeks using the surv_incentive display (two participants). We conducted a follow-on experiment by re-posting surv_qrcode_only for two more weeks (six participants) at which point we could no longer use the location. From video analysis we determined that three individuals likely scanned the QR code, but elected not to visit the URL. Of these three individuals, one was from the surv_incentive, one was from the surv_qrcode_only and one was from the follow-on no incentive condition. In our study $85 \%(15 / 18)$ of people that scanned a QR code proceeded to visit the website, however our results may not be representative of a larger population. Nine participants visited the URL in the surv_qrcode_only (plus five more in the follow-on), and only one in surv_incentive. This ratio suggests that the incentive may not have actually enticed the participants to scan the QR code. Moreover, more people scanned the poster in the follow-on than in surv_incentive, further re-enforcing the tendency to scan the no incentive condition.

Five participants, all in the surv_qrcode_only, started the survey. Of these five, one was under 18 and discarded, another selected "Prefer not to answer" and "Neutral" for every question. The remaining three participants were all students of age 18-24, two male and one female. Of these three, two completed the survey. Interestingly, one answered the question "How often do you scan a QR code?" with "Every time I see one" while the other answered "Rarely." The devices of the nine participants include four iPhones, four Android devices, and one BlackBerry.

\subsection{Limitations}

This experiment was conducted in a single, on-campus location, limiting results to a single population. The location was in the computer science building, leading 
to a relatively technologically-sophisticated population. The location was near a primary walking path and near a coffee shop, both of which contribute to a wider demographic, but the single location certainly has population bias. Further, the participant pool may have degraded between conditions, since the same location was used for both conditions. For example, participants may not scan a new poster, anticipating that the new poster is part of the same study.

Another technical limitation was the subjectivity in determining whether a person scanned the QR code. If a correlated entry appeared in the server logs, the person certainly scanned the QR code. However, without the server log entry, we are forced to decide whether the images indicate that a person scanned the QR code. As shown in the results, nearly all (85\%) of people who scanned the code also visited the website leaving $15 \%$ subject to scrutiny. None-the-less, the analysis is subjective and it is possible that some instances may have been misclassified.

\section{$5 \quad$ Security Implications}

Unsurprisingly, of the 229 participants we observed, more than $80 \%$ (184) used a mobile device in our studies. Contrary to national metrics from around the time of our study showing an Android majority [9] [4], we observed 57\% (105) using an Apple iOS device and 38\% (69) using an Android based device (the remaining five percent used Blackberry and Windows mobile devices). Given that the majority used either an iOS or Android device, it is also no surprise that of the mobile clients, $96 \%$ of browsers are WebKit based. Figure 11 shows user-agent distribution for the measured devices.

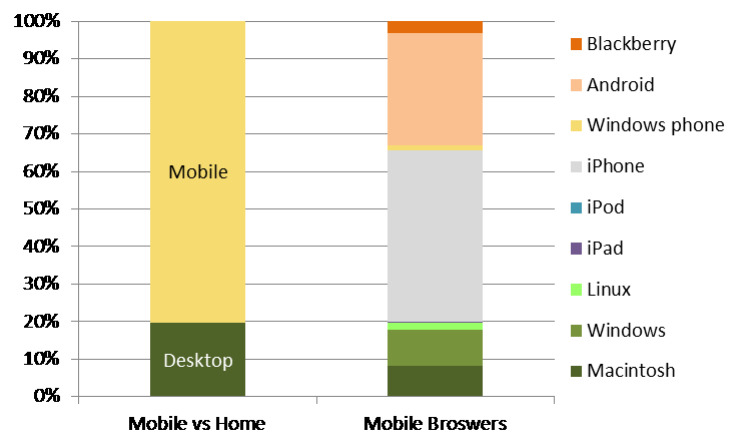

Fig. 11: Observed user-agent and mobile vs desktop browser distribution.

At the time of this research, known vulnerabilities and public exploits existed that target the WebKit browser directly [6] [1] or a content handler (such as PDF files [2]) for all Android and iOS devices observed in this study. In order to take advantage of such vulnerabilities, an attacker must persuade the user to visit a web site serving malicious content, possibly using a QR code. A successful 
attack will result in the attacker having remote access to the same resources as the browser.

In many cases, executing in the same context as the browser may be enough to achieve attack objectives such as reading browser cookies or stealing website passwords. Other attack objectives require more privileged access. This elevated access is often referred to as "rooting" or "jailbreaking" the mobile device. Rooting exploits are often very dependent on the operating system version.

We observed the operating system distribution for iOS and Android devices. The fragmentation present for Android is consistent with what has been reported in the literature and by Google $[7,18,30] .{ }^{1}$ We observed nine different iOS versions, but greater than $80 \%$ (89) of iOS devices were running the most recent, 5.0.1. In Android we observed 11 different iOS versions, and no single version was present on more than $30 \%$ of devices. The two versions with the highest percentage of devices were 2.3.3(18\%) and 2.3.4 (27\%), neither of which were the most current software within an Android branch at the time of the study (in this case 2.3.7). Rooting exploits not requiring physical device access were publicly available at the time [13] [3] for 83\% (59) of Android and 17\% (19) of iOS devices we observed.

Compared to the actions required from an unscrupulous attacker, conducting our study demanded significantly more effort. For example, we spent considerable time ensuring that flyers at all locations were available. Since each location required a unique flyer, we tracked which specific flyers were posted so that the distribution of conditions and specific URLs were maintained throughout the experiment. Similarly, our exposure was limited to locations where we were ethically and legally permitted to post QR codes. A would-be attacker does not have such problems, permitting the attacker to expend significantly less time and receive significantly more exposure when compared to this study. For this reason, our findings should be considered an extreme lower bound on the susceptibility of QRishing.

In many cases QRishing would be conducted physically, meaning that an attacker would have to find some way to post QR codes where a user might approach and scan the code. The physical format of QRishing could be realized in many forms: full posters, sticker overlays, etc. In our study we simply posted disingenuous flyers. When compared to digital forms of phishing, such as email, the cost of performing a QRishing attack is likely comparatively high. The cost of printing QR codes is negligible, but it takes time to post them and other risks, such as being physically caught placing a malicious QR code, represent considerable potential cost. On the other hand, an attacker, unbound by legal and ethical issues, can place QR codes in a wider range of places than we were permitted.

Mobile browsers are largely not employing technical controls that have been available in desktop browsers for some time. For instance, technical controls may be used to assist the user in making security-conscious decisions. Some reader applications already display the $\mathrm{QR}$ code content prior to performing an action,

\footnotetext{
${ }^{1}$ Google's self-reported numbers [7] are grouped less precisely than ours. But when we group ours accordingly, they align closely.
} 
such as visiting a website. While this simple action requires the user to "click one more button," the opportunity to at least assess the potential for a questionable domain is beneficial. This could be augmented with security-specific controls that are already ubiquitous in desktop browsers such as comparing the scanned URL to a blacklist or some other "safe browsing" technology.

Security indicators for valid certificates and SSL/TLS connections are widely adopted by desktop-browser vendors and allow consumers to assess the security of their communications over the web. However, the deployment of technical controls and security indicators to mobile browsers is complicated by the relatively small screen real estate for handheld devices. In an empirical study of ten mobile browsers and two tablet browsers, Amrutkar et al. find that many of the World Wide Web Consortium's (W3C) guidelines for security indicators in web user interfaces are not implemented on mobile browsers and that there is little consistency among mobile browsers that do implement security indicators [10].

Another technological control specific to smartphones is to enable timely application of security updates to mobile browsers and core device software. Feature updates could be separated from security updates. The separation would allow security updates to be applied quickly and independently of feature updates, allowing economic motivations to drive the release (or not) of feature updates. This control does not specifically address the threat of QR codes, but can mitigate the subsequent threats posted by the malicious websites. Similarly, if the browser was a self-contained component, similar to other mobile applications, it could be updated independent of the core software of the device. In this light, alternative browsers such as Firefox mobile, provide a method to use an updated browser on old devices where system software is no longer updated.

\section{Conclusion}

We presented two experiments demonstrating that $\mathrm{QR}$ codes are a viable method for conducting phishing attacks. We posted QR code posters across 139 different locations and found that 225 individuals scanned at least one poster over a fourweek period. Overall, $61 \%$ of the disingenuous posters were scanned by at least one person.

Most users (75\%) scanned the QR code out of curiosity or for fun. Comparatively, very few scanned in order to solicit more information about the context surrounding the QR code. The results of our surveillance experiment indicate that most users who scan a QR code will subsequently visit the related URL, even if the domain is unfamiliar and uses "URL shortener" style URLs. Providing security controls that already exist in desktop browsers to mobile browsers may foster safer behavior than what we observed in this study.

While a QRishing attack likely requires more resources than a typical email oriented phishing attack, the cost of conducting a QRishing attack is negligible. However, indirect costs, such as physically being caught, present considerable additional risk over traditional phishing mechanisms. None-the-less, if the attacker wishes to target a particular audience, such as smartphone users, QRishing may be a viable option. The ease with which such an attack can be mounted against 
current smartphones is particularly concerning given the long patching cycle and potential for an attacker to gain elevated privileges on the device. With or without the security-specific controls, user awareness of new threats like QRishing will be critical as mobile devices become increasingly popular.

\section{References}

1. About the security content of iOS 4.3. http://support.apple.com/kb/HT4564, Mar. 2011.

2. About the security content of iOS 5.0.1. http://support.apple.com/kb/HT5052, Nov. 2011.

3. CVE-2011-3874 - libsysutils rooting vulnerability (zergRush). http://code. google.com/p/android/issues/detail?id=21681, Nov. 2011.

4. Generation app: $62 \%$ of mobile users 25-34 own smartphones. http://blog. nielsen.com/, Nov. 2011.

5. The Male vs. Female Debate Goes Mobile. http://blog. compete.com, Nov. 2011.

6. Android bug opens devices to outside control: experts. http://www.reuters.com/ article/2012/02/24/us-google-android-security-idUSTRE81N1T120120224, Feb. 2012.

7. Android Developer Guide: Platform Versions (Feb 1, 2012). http://developer. android.com, Feb. 2012.

8. Cisco Visual Networking Index: Global Mobile Data Traffic Forecast Update 20112016. White Paper: http://www.cisco.com/en/US/solutions/collateral/ ns341/ns525/ns537/ns705/ns827/white_paper_c11-520862.html, Feb. 2012.

9. comScore Reports December 2011 U.S. Mobile Subscriber Market Share. http://www.comscore.com/Press_Events/Press_Releases/2012/2/comScore_ Reports_December_2011_U.S._Mobile_Subscriber_Market_Share, Feb. 2012.

10. C. Amrutkar, P. Traynor, and P. C. van Oorschot. An Empirical Evaluation of Security Indicators in Mobile Web Browsers. Technical Report GT-CS-11-10, Georgia Institute of Technology, 2011.

11. L. Borrett. Beware of Malicious QR Codes. http://www . abc.net.au/technology/ articles/2011/06/08/3238443.htm, June 2011.

12. U. C. Bureau. Pittsburgh census map. http://www.city.pittsburgh.pa.us/cp/ html/census_map.html, 2000.

13. chpwn, MuscleNerd, and chronicdevteam. iOS Jailbreaking Website. http:// jailbrea.kr/.

14. R. Dhamija and J. Tygar. The battle against phishing: Dynamic security skins. In Proceedings of SOUPS 2005, pages 77-88. ACM, 2005.

15. R. Dhamija, J. Tygar, and M. Hearst. Why phishing works. In SIGCHI conference on Human Factors in computing systems, pages 581-590. ACM, 2006.

16. J. Downs, M. Holbrook, and L. Cranor. Decision Strategies and Susceptibility to Phishing. In Proceedings of SOUPS 2006, pages 79-90. ACM, 2006.

17. S. Egelman, L. Cranor, and J. Hong. You've been warned: an empirical study of the effectiveness of web browser phishing warnings. In SIGCHI conference on Human factors in computing systems, pages 1065-1074. ACM, 2008.

18. A. Gahran. Why 'Android fragmentation' isn't so bad. http://www.cnn.com/ 2012/02/17/tech/mobile/android-fragmentation-gahran/, Feb. 2012.

19. J. Han, E. Owusu, T.-L. Nguyen, A. Perrig, and J. Zhang. ACComplice: Location Inference using Accelerometers on Smartphones. In Proceedings of the 4 th COMSNETS, Jan. 2012. 
20. M. Hara, M. Watabe, T. Nojiri, T. Nagaya, and Y. Uchiyama. Optically readable two-dimensional code and method and apparatus using the same, Mar. 101998. US Patent 5,726,435.

21. P. Kumaraguru, S. Sheng, A. Acquisti, L. Cranor, and J. Hong. Teaching Johnny not to fall for phish. ACM Transactions on Internet Technology, 10(2):7, 2010.

22. T. Moore and B. Edelman. Measuring the perpetrators and funders of typosquatting. Financial Cryptography and Data Security, pages 175-191, 2010.

23. A. Neumann, J. Barnickel, and U. Meyer. Security and privacy implications of url shortening services. In Proceedings of the Workshop on Web 2.0 Security and Privacy, 2010.

24. R. Newman. Consumer Alert: QR Code Safety. Better Business Bureau. http://sandiego.bbb.org/article/consumer-alert-qr-code-safety-28037, June 2011.

25. Office of Institutional Research and Analysis. Carnegie mellon factbook. http://www.cmu.edu/ira/factbook/pdf/facts2012/ entire-fb-for-web-as-of-3-1-121.pdf, Feb. 2012.

26. S. Radwanick. 14 Million Americans Scanned QR Codes on their Mobile Phones in june 2011. http://www. comscore.com, Aug. 2011.

27. S. Sheng, B. Magnien, P. Kumaraguru, A. Acquisti, L. Cranor, J. Hong, and E. Nunge. Anti-phishing phil: The design and evaluation of a game that teaches people not to fall for phish. In Proceedings of SOUPS 200\%. ACM, 2007.

28. C. Tamir. AVG (AU/NZ) Cautions: Beware of Malicious QR Codes. PCWorld. https://appsec-labs.com/blog/tag/qrcode, June 2011.

29. D. M. Todd. Security expert warns smartphone users of the risks in scanning cybercoding. http://www. post-gazette.com. Accessed, June 2012.

30. T. Vidas, D. Votipka, and N. Christin. All your droid are belong to us: A survey of current android attacks. In Proceedings of the 5th USENIX WOOT, pages 10-10. USENIX Association, 2011.

31. P. Wagenseil. Anti-anonymous hacker threatens to expose them. http://www. msnbc.msn.com. Accessed, Mar. 2012.

32. Y. Zhang, J. Hong, and L. Cranor. Cantina: a content-based approach to detecting phishing web sites. In Proceedings of the 16th international conference on World Wide Web, pages 639-648. ACM, 2007. 


\section{Appendix}

\begin{tabular}{|l|l|l|}
\hline No. & Application [Vendor] & $\begin{array}{l}\text { Auto } \\
\text { Visit }\end{array}$ \\
\hline 1 & Barcode Scanner [Versolab] & no \\
\hline 2 & $\begin{array}{l}\text { ShopSavvy (Barcode and QR Scan- } \\
\text { ner) [ShopSavvy, Inc. }\end{array}$ & yes \\
\hline 3 & $\begin{array}{l}\text { RedLaser Barcode and QR Scanner } \\
\text { [eBay, Inc. }\end{array}$ & no \\
\hline 4 & $\begin{array}{l}\text { ScanLife Barcode and QR Reader } \\
\text { [Scanbuy, Inc.] }\end{array}$ & yes \\
\hline 5 & AT\&T Code Scanner [AT\&T Inc] & no \\
\hline 6 & $\begin{array}{l}\text { pic2shop - Barcode Scanner } \\
\text { [Vision Smarts] }\end{array}$ & no \\
\hline 7 & $\begin{array}{l}\text { Bakodo - Barcode Scanner } \\
\text { [Dedoware, Inc] }\end{array}$ & no \\
\hline 8 & $\begin{array}{l}\text { NeoReader - QR reader } \\
\text { [NeoMedia Technologies, Inc] }\end{array}$ & yes \\
\hline 9 & $\begin{array}{l}\text { i-nigma QR Code scanner } \\
{[3 G \text { ision] }}\end{array}$ & yes \\
\hline 10 & $\begin{array}{l}\text { MOBILETAG - Barcode Scanner } \\
\text { [Mobile Tag] }\end{array}$ & yes \\
\hline
\end{tabular}

(a) iOS Applications

\begin{tabular}{|c|c|c|}
\hline No. & Application [Vendor] & $\begin{array}{l}\text { Auto } \\
\text { Visit }\end{array}$ \\
\hline 1 & Barcode Scanner [ZXing] & no \\
\hline 2 & $\begin{array}{l}\text { ShopSavvy Barcode Scanner } \\
\text { [ShopSavvy, Inc.] }\end{array}$ & yes \\
\hline 3 & $\begin{array}{l}\text { QuickMark Barcode Scanner } \\
\text { [SimpleAct, Inc.] }\end{array}$ & no \\
\hline 4 & $\begin{array}{l}\text { RedLaser Barcode and QR Reader } \\
\text { [eBay Mobile] }\end{array}$ & no \\
\hline 5 & $\begin{array}{l}\text { ScanLife Barcode and QR Reader } \\
\text { [Scanbuy, Inc.] }\end{array}$ & yes \\
\hline 6 & Barcode scanner [george android] & no \\
\hline 7 & $\begin{array}{l}\text { i-nigma Barcode Scanner } \\
\text { [3G Vision] }\end{array}$ & yes \\
\hline 8 & $\begin{array}{l}\text { AT\&T Code Scanner } \\
\text { [AT\&T Service, Inc.] }\end{array}$ & no \\
\hline 9 & $\begin{array}{l}\text { ixMAT Barcode Scanner } \\
\text { [ixellence.com] }\end{array}$ & no \\
\hline 10 & $\begin{array}{l}\text { BARCODE SCANNER } \\
\text { [Jet Ho] }\end{array}$ & no \\
\hline
\end{tabular}

(b) Android Applications

Table 1: QR code reader applications tested. Five of the top ten free iOS applications and three of the top ten free Android applications automatically visit URLs scanned from QR codes.

Many mobile devices do not have any QR code reading software pre-installed. Tables 1(a) and 1(b) show the specific applications tested, whether the application automatically visits a URL retrieved from a barcode, and the order (top to bottom) of popularity on March 8, 2012. Several of the most popular iOS applications were either not free, or did not scan QR codes. We did not test any applications that were not free. Thirty percent of these top ten free scanning applications in the Google Play Market and 50\% in the Apple App Store immediately visit a scanned URL in the default configuration. When applications employ this feature, the user has no opportunity to visually inspect the URL prior to visiting that URL. 